\title{
Intereses investigativos de estudiantes de la Licenciatura en Educación Básica en la modalidad a distancia
}

\author{
Sandra Lucía Guerra-Gómez
}

\begin{abstract}
Resumen
El presente artículo tiene como propósito compartir parte de los resultados de una investigación, cuyo propósito fue sistematizar algunos aspectos relacionados con los proyectos de grado desarrollados por los estudiantes de la Licenciatura en Educación Básica con énfasis en Matemáticas, Humanidades y Lengua Castellana de la Universidad Pedagógica y Tecnológica de Colombia (UPTC), durante el lapso de 2008 a 2011. La metodología desarrollada fue de tipo documental con finalidades informativas. En esta se recolectó información a través de un archivo generado, a partir del rastreo de las investigaciones de los estudiantes que, a su vez, permitió determinar sus intereses investigativos, las líneas de investigación, las asignaturas y temas de mayor escogencia para realizar las investigaciones de correspondencia con la ubicación de los estudiantes en los diferentes Centros Regionales de Educación a Distancia. Se usó como estrategia la creación de una base de datos con la información recopilada, a partir de la cual se generaron datos cualitativos y cuantitativos que fueron sistematizados e interpretados para ser presentados ante la comunidad académica. Se pudo concluir que los estudiantes optan por investigar en la asignatura de lengua castellana debido al interés en solucionar problemas de aprendizaje en lectura y escritura a poblaciones a las cuales estaba dirigida la investigación y las inscribieron dentro de la línea Aula y escuela. Esto se debe también a que es una línea que pretende desarrollar procesos de aprendizaje a partir de las problemáticas encontradas en los contextos educativos donde hace extensión la Licenciatura en Educación Básica.
\end{abstract}

Palabras clave: asignaturas, intereses investigativos, línea de investigación, reflexión, sistematización.

\section{Research Interests Among Students of the Distance-Learning Degree in Basic Education}

\begin{abstract}
This article shares part of the results of a research study that aimed to systematize certain aspects of the final projects undertaken by students in the Degree of Basic Education with an emphasis on Mathematics, Humanities and the Spanish Language at the Universidad Pedagógica y Tecnológica de Colombia (UPTC) from 2008 to 2011. The study used a documentary-style methodology for informational purposes. Information was collected through a generated file, which was created based on students' research work, thereby making it possible to determine the research interests, areas, and most commonly chosen subjects and topics for research, and correlate these with students' locations in different regional centers of distance education. The strategy involved creating a database with the information collected. From this, qualitative and quantitative data was generated, then systematized and interpreted for presentation to the academic community. It could be concluded that students choose to conduct research in the Spanish language due to an interest in solving reading and writing learning problems among the populations that the research is based on. These projects were registered in the research area titled Classroom and School. The projects were also chosen because this research area aims to develop learning processes based on problems found in educational contexts where university extension activities are undertaken in the Basic Education Degree.
\end{abstract}

Keywords: subjects, research interests, area of research, reflection, systematization.

* Magíster en Educación. Profesora de la Facultad de Estudios a Distancia e investigadora del grupo SIEK, Universidad Pedagógica y Tecnológica de Colombia, Tunja, Colombia.

Correo electrónico:

sandra.guerra@uptc.edu.co

Recibido: 10 de marzo del 2014

Aprobado: 6 de noviembre del 2014

Cómo citar este artículo: Guerra-Gómez, Sandra Lucía. "Intereses investigativos de estudiantes de la Licenciatura en Educación Básica en la modalidad a distancia". Rastros Rostros 16.30 (2014): 77-87. Impreso. doi: http://dx.doi.org/10.16925/ra.v16i30.822
Interesses de pesquisa de estudantes da Licenciatura em Educação Básica na modalidade a distância

\section{Resumo}

O presente artigo tem como propósito compartilhar parte dos resultados de uma pesquisa, cujo propósito foi sistematizar alguns aspectos relacionados com os projetos de graduação desenvolvidos pelos estudantes da Licenciatura em Educação Básica (até a $8^{\mathrm{a}}$ série) com ênfase em Matemática, Humanidades e Língua Castelhana, na Universidad Pedagógica y Tecnológica de Colombia (UPTC), no período de 2008 a 2011. A metodologia desenvolvida foi de tipo documental com finalidades informativas. Nela coletou-se informação através de um arquivo gerado a partir do rastreamento das pesquisas desses estudantes que, por sua vez, permitiu determinar seus interesses de pesquisa, as linhas de pesquisa, as matérias e os temas mais escolhidos para realizar as pesquisas de correspondência com a localização dos estudantes nos diferentes Centros Regionais de Educação a Distância. Usou-se como estratégia a criação de uma base de dados com a informação copilada, a partir da qual se geraram dados qualitativos e quantitativos que foram sistematizados e interpretados para serem apresentados ante a comunidade acadêmica. Pôde-se concluir que os estudantes optam por pesquisar na matéria de língua castelhana devido ao interesse em solucionar problemas de aprendizagem em leitura e escrita a populações às quais estava dirigida a pesquisa e as escreveram dentro da linha de pesquisa Classe e Escola. Isso se deve também a que é uma linha que pretende desenvolver processos de aprendizagem a partir das problemáticas encontradas nos contextos educativos em que se faz extensão a Licenciatura em Educação Básica.

Palavras-chave: matérias, interesses de pesquisa, linha de pesquisa, reflexão, sistematização. 


\section{Introducción}

El presente artículo pretende mostrar los resultados de un trabajo de exploración desarrollado sobre los intereses investigativos de los estudiantes de la Licenciatura en Educación Básica con énfasis en Matemáticas, Humanidades y Lengua Castellana, programa ofrecido por la Facultad de Estudios a Distancia (FESAD) de la Universidad Pedagógica y Tecnológica de Colombia (UPTC). Este trabajo surgió por el interés de algunos integrantes del Grupo Saberes Interdisciplinares en Construcción (SIEK), que originó un proyecto de investigación, denominado "Sistematización de los Proyectos del Programa en Educación Básica 2008-2011", el cual fue presentado ante el Centro de Investigaciones de Educación a Distancia (CIDEA) de la FESAD y con un registro con código sGi ante la Dirección de Investigaciones de la UPTC.

Este trabajo surgió con la intención de ir tras las huellas de la investigación de los trabajos de grado desarrollados por los estudiantes de la licenciatura, a fin de crear un banco de datos con información sobre sus investigaciones, para que así, de modo fácil y de acceso rápido, pudieran ser consultadas por la comunidad académica. De igual manera, con el fin de tener una evidencia tangible de los procesos de autoevaluación y acreditación del programa, la cual señala dentro de sus factores el reconocimiento que se debe tener de los trabajos realizados por los estudiantes a su paso por la universidad. Este tipo de información solo se encuentra directamente en la biblioteca de la universidad y en algunos CREAD, por cuanto no se cuenta con el acceso a estos documentos en la red y no existen convenios que presten servicios como el que ofrece la Red Nacional de Bibliotecas Públicas, la cual proporciona el servicio de bibliotecarios a nivel nacional, regional, departamental y local, y que se convierte en un aspecto que puede fortalecer la investigación que realizan los estudiantes que se encuentran ubicados en cualquiera de los centros de educación a distancia. Por esta razón, se considera que la información compilada puede llegar a facilitar la búsqueda de los trabajos de grado desarrollados por los estudiantes y que merecen ser retomados y considerados como antecedentes para las próximas investigaciones por parte de sus docentes y compañeros de profesión. Asimismo, se desea generar reflexiones en los futuros docentes en el sentido de que los maestros, hoy más que nunca y a partir de una mirada crítica a su práctica pedagógica, asuman el compromiso de reali- zar cambios significativos que ayuden a transformar la realidad escolar y contribuyen a mejorar la calidad de la educación (Sánchez 15).

Pero, ¿por qué investigar sobre los trabajos de grado de los estudiantes de modalidad a distancia? Para contextualizar la investigación, se determinó en su momento investigar sobre la trascendencia que tiene un trabajo de grado y se entiende que este consiste en la formulación, planeación y, en algunos casos, ejecución de un proyecto en el que el estudiante de pregrado pone en práctica los conocimientos adquiridos en el transcurso del programa académico. De la misma manera, se entiende que este tipo de trabajos deben poseer un componente investigativo que "acentúa la interpretación y la comprensión de la realidad educativa desde los significados de las personas involucradas y estudian sus intenciones, creencias, motivaciones y otras características no directamente manifiestas ni susceptibles de experimentación" (Arnal, Rincón y Latorre 40). Es así como la investigación se convierte en una oportunidad para que se fundamenten, apliquen y se produzcan conocimientos que conlleven al desarrollo de habilidades investigativas con los saberes y competencias adquiridas a través de su formación académica y a partir de los cuales se planteen soluciones a los problemas de su contexto social y laboral. Por tanto, se considera que la investigación en la modalidad a distancia ofrece una oportunidad de repensar en las problemáticas educativas y formativas que se presentan en los diversos contextos en los que llega una licenciatura. Por esta razón, se hace interesante que, además de ser una modalidad diferente a la presencial, ofrece las mismas oportunidades de desarrollar procesos investigativos con calidad.

Dentro de las modalidades de trabajo de grado existentes en el programa de la UPTC, y estipuladas en la Resolución 36 de 2010, se encuentra el trabajo de investigación en educación que posee un carácter formativo y la monografía de tipo teórico o práctico en la que se resuelve una hipótesis de trabajo para describir una situación problémica, analizarla y establecer causas, consecuencias y soluciones. En ambos se siguen los pasos de una investigación formativa, en los cuales se deben contemplar las partes de un proyecto como lo son los objetivos, preguntas de investigación, hipótesis (si aplica en la investigación) marco teórico o conceptual, metodología, resultados y discusión y conclusiones.

Lo anterior permite justificar que los procesos investigativos se pueden aplicar también a la investigación en la modalidad a distancia, a pesar de tener a los estudiantes en lugares alejados de una sede central. Una 
de las características más importantes de la educación a través de esta modalidad es la implicación permanente de estudio, la objetivación del saber, el que se deba aprender a aprender de una manera constante, en una educación socializante, pues exige del estudiante responsabilidad, integralidad y compromiso. Esto lleva a su vez al establecimiento de relaciones interpersonales, a que exista trabajo cooperativo, colaborativo, así como también a que los estudiantes se planteen metas de aprendizaje sobre lo que desea aprender, en especial a autorregular el logro de sus aprendizajes. La modalidad de educación a distancia es una oportunidad para superar las limitaciones geográficas, los costos elevados y las discriminaciones sociales de la educación, así como también cuando uno se asume como educador lo hace como un ser de relación y la pedagogía se ocupa del sentido de esa relación (Gutiérrez y Prieto 5, 6).

\section{Metodología}

Los estudiantes de la modalidad a distancia tienen la particularidad de asistir quincenalmente a encuentros presenciales, se encuentran matriculados en 23 CREAD, distribuidos en siete departamentos. En su mayoría poseen una edad de entre 18 y 40 años. Algunos de ellos han terminado sus estudios en escuelas normales, que son instituciones educativas encargadas de la formación de los maestros de escuela. Estos normalistas se desempeñan como docentes en instituciones educativas rurales o urbanas, y varios de ellos se encuentran ejerciendo como docentes. La mayoría posee un computador personal para la realización de sus trabajos, y algunos de ellos poseen conectividad en sus escuelas o, en casos particulares, se dirigen a un café internet. Por lo tanto, hace novedosa la investigación el querer conocer, analizar y reflexionar sobre los trabajos de grado desarrollados por los estudiantes desde el contexto en el que se encontraron en el momento de realizar trabajos de grado. A su vez, saber que se tienen estudiantes en 23 lugares diferentes a la sede central de Tunja hace que los investigadores del grupo siek se interesen en tener un acercamiento a lo que se investiga en los diferentes lugares del país.

\section{Importancia de la sistematización y de la investigación}

La investigación desarrollada tuvo como referente principal la sistematización de experiencias, estrategia que es entendida como esa voz que permite que los saberes de prácticas y sus conocimientos emerjan y vayan a la sociedad con fuerza propia para disputar un lugar en los terrenos del saber y el conocimiento, como un escenario más de las resistencias y una forma de lucha social. Así, Mejía y Bravo conciben la sistematización como "un desarrollo local del saber contextualizado" (133).

Torres, dentro de sus reflexiones frente a la sistematización de las prácticas educativas, indica que, al sistematizar, se "aspira a reconstruir la totalidad concreta que constituye cada práctica social privilegiando los significados que los actores le atribuyen y que constituyen un campo de fuerzas que configura la identidad de los grupos y las organizaciones que la impulsan" (4). Se puede relacionar entonces con el establecimiento de un orden que tiene por objetivo obtener los mejores resultados posibles de acuerdo con el fin que se tenga que alcanzar. La sistematización se puede aplicar en los ámbitos científicos y académicos, pero también hay muchas situaciones de la vida cotidiana que implican cierto procesamiento de datos para lograr un objetivo específico. En este sentido, un acto tan sencillo como usar el diario de campo en el aula, dentro de nuestro rol como maestros, es, sin duda alguna, un modo de sistematizar el uso de nuestro tiempo de acuerdo con nuestras necesidades y preferencias. Este permite revisar nuestras debilidades, oportunidades, y fortalezas y llegar así a un acercamiento a las soluciones que podemos dar en las diversas situaciones que se generen regularmente con los procesos de enseñanza y aprendizaje de nuestros niños.

Ahora bien, en cuanto a trabajos desarrollados en el campo de sistematización de experiencias a nivel nacional, se tiene conocimiento de un trabajo de investigación, denominado "Tendencias investigativas en educación y pedagogía: estudio de las regiones investigativas de la Maestría en Educación de la uis" que pretendió mostrar "las formas cómo se realiza la investigación en educación y pedagogía en la Maestría de Pedagogía de la uis, como un compromiso con esta pretensión comprensiva, desvelando, de paso la manera como se abordan los procesos de investigación en esa maestría" (Murcia, Murcia y Urbina 71-98). Este artículo es importante por cuanto investigó y reflexionó en torno a los diferentes escenarios y a la población que se encuentra en situación de vulnerabilidad a nivel nacional. Esto posibilita revisar lo que está sucediendo a nivel de los 23 CREAD a los que ha llegado la licenciatura y cómo los estudiantes que allí se encuentran tienen interés por dar solución a las problemáticas allí vivenciadas. De igual manera, se tiene conocimiento 
del trabajo "Lo que investigan estudiantes de maestría en educación: Análisis desde un estado del arte", cuya autora muestra que "los resultados de su investigación enmarcados dentro de la línea de investigación Evaluación escolar y desarrollo educativo regional de la Maestría en Educación de la Universidad Pedagógica Nacional de Bogotá" (García 9-14). Este estudio reveló que "los trabajos de investigación que se expresan en las tesis de grado constituyen una riqueza valiosa en la construcción de conocimiento especializado sobre diferentes aspectos de la educación", y, por tanto, se constituye como un argumento valioso para que otras personas conozcan y valoren lo que los estudiantes de la modalidad a distancia pueden aportar con sus investigaciones.

En consecuencia, basados en estos casos especiales, particulares y pertinentes para la investigación, se consideró de suma importancia realizar una investigación a nivel local, con el fin de dar a conocer los procesos investigativos que se han venido adelantando y, a su vez, para que puedan desarrollar nuevas intervenciones en el aula de manera innovadora, creativa, con rigurosidad, claridad y con formación científica.

El Proyecto académico educativo (PAE) de la Licenciatura contempla lo siguiente:

La investigación en el Programa de Licenciatura en Educación Básica con énfasis en Matemáticas, Humanidades y Lengua Castellana, se articula con la formación pedagógica y se relaciona con la integralidad del conocimiento, a fin de hacer realidad los enfoques transdisciplinarios para desarrollar la multidimensionalidad del ser humano. En esa perspectiva, se establecen como líneas de investigación: Aula y Escuela y Desarrollo Educativo y Social. (89-109)

Lo anterior se toma como base para la clasificación de los trabajos de grado de los estudiantes de la licenciatura, y poder así realizar un reordenamiento de la información que se encuentra consolidada en los documentos que reposan en el grupo sIEK.

Sobre la investigación que realizan los estudiantes de la modalidad a distancia, se puede decir que tiene un carácter formativo. Esto crea el compromiso de conocer una comunidad educativa por parte de todos y se concreta en una función jerarquizante y legitimadora, que conlleva a diferenciar a los que se encuentran involucrados en el proceso de formación de investigadores: "Maestros, docentes investigadores, semilleros de investigación y tutores, en las relaciones jerárquica donde el tutor o maestro investigador, representado como un adulto experto, sostiene el crecimiento e incubamiento del saber en sus semilleros" (Messina 61-75).

De acuerdo con lo anterior y de conformidad con el verdadero sentido que tiene la formación para la investigación, se puede especificar que dicha formación es un proceso que implica prácticas y actores diversos, en el que la intervención de los formadores como mediadores humanos se concreta en un quehacer académico consistente en promover y facilitar, de manera sistematizada, el acceso a los conocimientos, el desarrollo de habilidades, hábitos y actitudes y la internalización de valores, que demanda la realización de la práctica denominada investigación (Moreno 521).

Frente a los procesos investigativos, se debe revisar lo que se está desarrollando al interior del programa de Licenciatura en Educación Básica adscrito a la modalidad a distancia, tanto por parte de los docentes que asesoran a sus estudiantes como de los resultados que se muestran producto de un proceso investigativo que se rige por las normativas establecidas por la universidad. Dentro de estas, se encuentra la Resolución 36 de 2010 que establece la modalidad de trabajo de grado para los estudiantes de educación a distancia dentro de otras opciones, como las monografías y los trabajos de investigación propiamente dichos.

\section{Ruta metodológica}

Teniendo presente que esta investigación surgió como propuesta para establecer los antecedentes que se tienen de procesos investigativos desarrollados al interior del programa de Licenciatura en Educación Básica orientada en una modalidad de educación a distancia, se hace claridad que a nivel local no se tienen registros de investigaciones realizadas en este aspecto, por lo que la hace una investigación innovadora y pertinente para los procesos que al interior del programa y de la universidad se vienen adelantando.

La propuesta se basó en una investigación de tipo documental con finalidades informativas, por cuanto se basa en una panorámica acerca de la información que ha sido relevante y que el investigador ha tomado de diversas fuentes con el fin de organizarla, seleccionarla, analizarla y sintetizar las ideas y, así, finalmente presentarlas en un reporte final para darlo a conocer a la comunidad educativa. Al respecto, Vélez afirma que este tipo de investigación tiene como objetivo:

el desarrollo de las capacidades reflexivas y críticas a través del análisis, interpretación y confrontación de la información manejada. Entre los posibles propósitos de 
este tipo de investigación se encuentran: describir, mostrar, probar, persuadir o recomendar. La investigación debe llevar a resultados originales y de interés para el grupo social de la investigación. (12)

En particular, este tipo de investigación documental permite que los investigadores se adentren a reconocer, construir, explicar o comprender los diversos fenómenos educativos, pues se convierte, a la vez, en un proceso de aprendizaje para quienes participan de esta. Este proceso se encuentra con una acción informada y reflexiva sobre la propia práctica, en la búsqueda de explicaciones o interpretaciones, que han permitido la cualificación y el perfeccionamiento de los ejercicios investigativos y educativos.

Este proceso se dividió en dos fases: en la primera se procedió a la búsqueda y recopilación de la información, y la segunda fue subdividida en varias etapas: la clasificación, categorización, análisis y presentación de resultados.

Primera fase: para la compilación de información, se procedió listar los estudiantes graduados durante el lapso de 2008-2011. Dentro de estos resultados, se encontró que, durante el 2008, se graduaron 331 estudiantes, que estuvieron distribuidos en cuatro ceremonias celebradas en abril, julio, septiembre y diciembre. En el 2009, se graduaron 522 durante los mismos meses. En el 2010, se graduaron 191 estudiantes, y 272 en el 2011. Con una totalidad de 1316 estudiantes (tablas 1 y 2), se procedió a realizar el rastreo de trabajos de investigación en la Biblioteca Central Jorge Palacios Preciado en Tunja.

Tabla 1. Relación de estudiantes graduados del programa por año

\begin{tabular}{lccccc}
\hline & $\mathbf{2 0 0 8}$ & $\mathbf{2 0 0 9}$ & $\mathbf{2 0 1 0}$ & $\mathbf{2 0 1 1}$ & Total \\
\hline Abril & 44 & 126 & 0 & 72 & 242 \\
Julio & 202 & 87 & 77 & 55 & 421 \\
Septiembre & 13 & 87 & 45 & 54 & 199 \\
Diciembre & 72 & 222 & 69 & 91 & 454 \\
\hline Total & 331 & 522 & 191 & 272 & $\mathbf{1 3 1 6}$ \\
\hline
\end{tabular}

Fuente: elaboración propia.

Tabla 2. Registro de información de trabajos de investigación
Segunda fase: se realizó la lectura del material, se ubicaron las principales ideas y se evaluó la calidad del material recabado. Asimismo, se delimitaron los temas, entre otros como nombre de autor(es), CREAD, tipo de investigación, resumen, población, resultados, conclusiones, de tal manera que se intervino su aspecto formal y su complejidad. Se realizó el inventario de la información recolectada. De esta manera, durante esta fase, se utilizó como técnica de recolección de información, el resumen analítico del escrito (RAE). Este consiste en realizar una ficha que se puede utilizar para resumir diferentes tipos de texto. Además, se aplicó especialmente para la elaboración de la sistematización de los trabajos realizados por los estudiantes y el posterior análisis de la información.

Se elaboraron fichas de contenido y, con base en la normatividad vigente, se tuvieron presentes las modalidades de trabajo de grado y las líneas de investigación. Para la presentación del informe final, se realizó un texto escrito, presentado ante el CIDEA de la FESAD, como requisito de investigación y evidencia para el grupo siek. De igual manera, se atiende a uno de los objetivos del proyecto el cual se propone comunicar con la mayor claridad y coherencia posibles los resultados, descubrimientos, comprobaciones o reflexiones logradas a través de todo el proceso de la investigación documental.

\section{Hallazgos}

De la información registrada: a partir de la búsqueda de cada trabajo de investigación en la Biblioteca Central de la Universidad y en la Facultad de Estudios a Distancia, se procedió a registrar la información encontrada en la tabla 2.

Con esta información, además de definir los trabajos de grado existentes entre 2008 y 2011, se procedió a realizar la consolidación en una base de datos con los principales componentes que la constituyen, para facilitar de esta manera la consulta en el momento que se requiera y por cualquier integrante de la comunidad educativa. De igual manera, reposa al interior del grupo SIEK con copia en el CIDEA y en la Dirección de Investigaciones (DIN), como requerimientos de la finalización del trabajo investigativo.

\begin{tabular}{l|c|c|c|c|c|c|c}
\hline Autor/Autores & $\begin{array}{c}\text { Título del } \\
\text { trabajo }\end{array}$ & Año & CREAD & Resumen & Palabras clave & $\begin{array}{c}\text { Línea de } \\
\text { investigación }\end{array}$ & $\begin{array}{c}\text { Tipo de } \\
\text { investigación }\end{array}$ \\
\hline & & & & & & & \\
\hline
\end{tabular}

Fuente: elaboración propia. 
De los CREAD, en el año 2008, se presentaron 197 trabajos de investigación de los cuales el $27 \%$ del total pertenecieron al CREAD de Sogamoso, un 23\% a Tunja, 9\% Yopal, 9\% Chiquinquirá, 8\% Bogotá, 8\% Cogua, 5\% Paz de Río, 4\% Barrancabermeja, 4\% Soatá, 1\% Acacías, $1 \%$ Fusagasugá y $1 \%$ Chiscas.

Para el 2009, el mayor número de trabajos de investigación se realizaron en el CREAD Tunja con un 51\% del total, le sigue Sogamoso con el 25\%, Yopal con el $11 \%$, Fusagasugá 3\%, Quetame, Duitama, Chiquinquirá, y Cogua con el $2 \%$ cada uno, Bogotá y Barrancabermeja con el $1 \%$ respectivamente, de un total de 132 trabajos analizados.

Para el 2010, Yopal obtuvo un 30\% del total de los trabajos analizados, el CREAD Tunja con el 22\%, Sogamoso un 17\%, Duitama un 6\%, Quetame el 6\%, Chiquinquirá el $5 \%$, Barrancominas un $4 \%$, Bogotá un $3 \%$, Fusagasugá un 3\%, y Guicán, Granada, Cogua y Acacías contaron con $1 \%$ respectivamente.

Para el 2011, de los 115 trabajos analizados, la mayoría fueron del CREAD de Tunja con un 28\%; del CREAD de Bogotá con el 17\%; de Chiquinquirá con el $14 \%$; de Quetame con el 7\%; de Sogamoso con el 5\%; de Cogua con el 5\%; de Yopal con el 4\%; de Duitama con el 4\%; de Guicán con el 4\%; de Acacías con el 3\%; de Soatá con el 3\%; de Fusagasugá con el 3\%, Barrancabermeja $1 \%$, Garagoa $1 \%$ y Gachetá el $1 \%$.

De la población seleccionada en las investigaciones: la población objeto de estudio de las investigaciones realizadas por los estudiantes de la Licenciatura en Educación Básica, adscritos a la modalidad a distancia, en su mayoría, estuvo conformada por niños que presentan ciertos problemas en el aprendizaje en las diferentes áreas del saber, en especial en las áreas de lectura, escritura y matemáticas; fueron niños vinculados a los diferentes grados de educación básica primaria y secundaria, con un rango de edad de entre 7 y 17. Se estima que las investigaciones realizadas por los estudiantes del programa comprendían poblaciones representativas teniendo en cuenta cada uno de los trabajos de grado realizados, a los que se trató de dar soluciones concretas a los diferentes problemas planteados. Por otra parte, fueron vinculados docentes y padres de familia, los cuales contribuyeron con información relevante en los procesos investigativos. En pocos casos, la población también estuvo conformada por adultos de ambos sexos, padres de familia, dedicados a diferentes actividades como la minería, jornaleros, albañiles y conductores entre otros. Estos tenían un bajo nivel educativo, puesto que en su mayoría cursaron hasta quinto de primaria.
De la línea de investigación: la línea de investigación que predominó en las investigaciones de los estudiantes fue Aula y Escuela. El objetivo, establecido en el programa, es:

Contemplar los distintos ejes de acción que se presentan en el contexto educativo real de sus estudiantes, en la cual el docente a actualizar sus conocimientos, a perfeccionar su acciones y desde luego a evaluar constantemente el acto educativo a través del gran marco como lo es la educación. (89)

Dentro de estos trabajos había algunos relacionados con las innovaciones pedagógicas, en su mayoría presentadas en el 2008, trabajos relacionados con educación, didáctica y escuela nueva. Los trabajos de innovación pedagógica se basaron en la aplicación de propuestas creativas desarrolladas al interior del aula, las cuales generaron un cambio intencional y controlado dentro de un proceso de construcción social entorno a un ambiente educativo dentro del aula, para que la comunidad educativa, en especial los docentes, tomaran consciencia de los diferentes problemas o fenómenos encontrado en los niños. A partir de estos trabajos de intervención, se generaron reflexiones y explicaciones que dieron lugar a cambios de estrategias que fueron adoptadas por los docentes, lo que mostró resultados positivos y favorables en las instituciones en las que fueron aplicados.

Respecto a lo anterior, surge como reflexión que al investigar dentro del aula, los docentes indagan, analizan y reflexionan sobre su propia práctica. Esto está ligado a una constante preocupación sobre el desarrollo de los distintos aspectos que componen cada uno de los contextos en los que se encuentran los estudiantes, teniendo en cuenta que hacen parte de diferentes culturas y que interactúan en el espacio escolar, al igual que se establecen diferencias en los aspectos académicos, pedagógicos, sociales, en lo escolar, el conocimiento y desconocimiento de lo propio, los problemas de aprendizaje y de enseñanza y cuya comprensión de los intereses particulares pueden ayudar a clarificar el conjunto de factores que condicionan los procesos de enseñanza-aprendizaje, y que frecuentemente se han incluido sin llegar a ser registrados y mucho menos socializados ante una comunidad educativa. Se reflexiona en torno a la investigación al interior del aula, evidenciándose la indagación, el análisis y la reflexión sobre su propia práctica; esto está ligado a una constante preocupación sobre el desarrollo de los distintos aspectos que 
componen cada uno de los contextos en los que se encuentran los estudiantes, sin olvidar que hacen parte de diferentes culturas y que interactúan en el espacio escolar. De igual manera, se establecen diferencias entre los aspectos académicos, pedagógicos, sociales, en lo escolar, el conocimiento y desconocimiento de lo propio. Esto sumado a los problemas de aprendizaje y de enseñanza y cuya comprensión de los intereses particulares pueden ayudar a clarificar el conjunto de factores que frecuentemente se han incluido sin llegar a ser registrados y mucho menos socializados ante una comunidad educativa. De esta forma, al investigar en el aula y la escuela como un espacio donde se recogen tantos aspectos diferentes es necesario resignificar el rol del docente pensando en forma permanente sobre las nuevas generaciones y que los registros que se hagan al respecto, sean la base para demostrar el trabajo arduo que tiene un docente que es investigador y que se preocupa por los cambios significativos, de tal manera que se alcancen hechos y resultados como la calidad educativa $\mathrm{y}$, por tanto, institucional.

Pasando a la línea de investigación Desarrollo Educativo y Social, se encontraron trabajos que comparten temáticas relacionadas con la educación rural, las realidades locales, regionales y nacionales, la etnoeducación y los conflictos sociales. El objetivo de esta línea, dentro de lo estipulado por el programa, es "dinamizar procesos de aprendizaje que accionen desde la reflexión un horizonte de interpretación y proposición frente al panorama educativo y social donde hace presencia el Programa en Educación Básica" (Proyecto Académico Educativo [PAE] 89-109).

Frente a lo anterior, aunque el porcentaje de trabajos adscritos a esta línea fue menor, plantea que los procesos educativos se realizan pensando en la cultura, en los problemas regionales y los procesos de formación democrática al interior de la escuela, las conductas sociales, colaborativas y cooperativas; estos procesos también tienen en cuenta las estrategias de intervención para generar alternativas pedagógicas y didácticas que fomenten en el aula de clase el respeto, así como la formación y desarrollo de comportamientos autónomos y responsables. También se reflexiona frente al interés por el fomento y desarrollo de diversos espacios para la cooperación y la convivencia ciudadanas.

A continuación, en las figuras 1, 2, 3 y 4, se presentan los resultados estadísticos por año de la inscripción de los trabajos a las líneas de investigación.

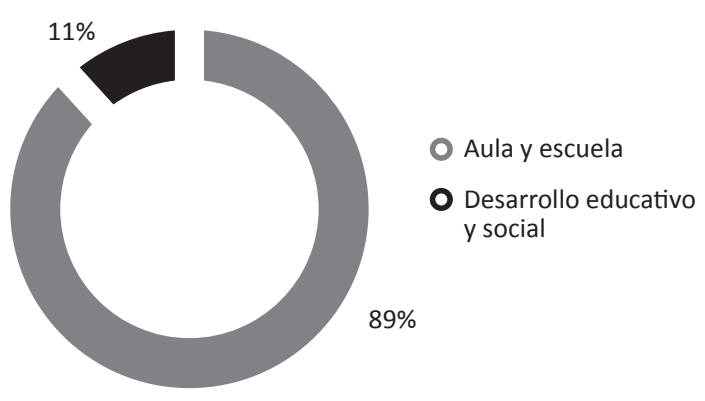

Figura 1. Trabajos de grado del 2008 por líneas de investigación. Elaboración propia.

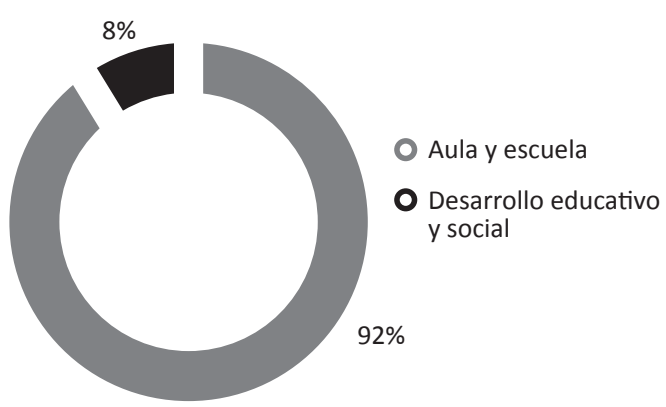

Figura 2. Trabajos de grado del 2009 por líneas de investigación. Elaboración propia.

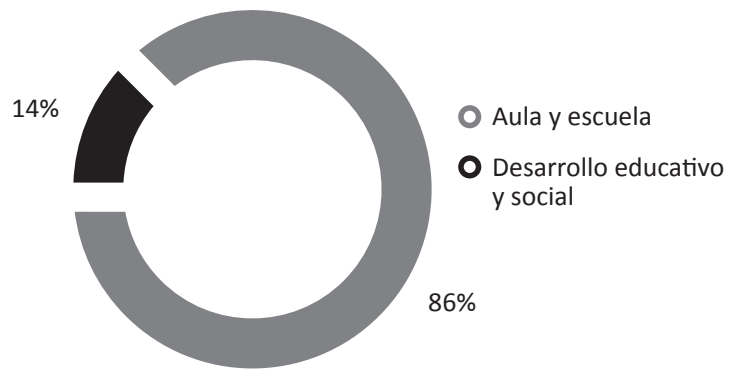

Figura 3. Trabajos de grado del 2010 por líneas de investigación. Elaboración propia.

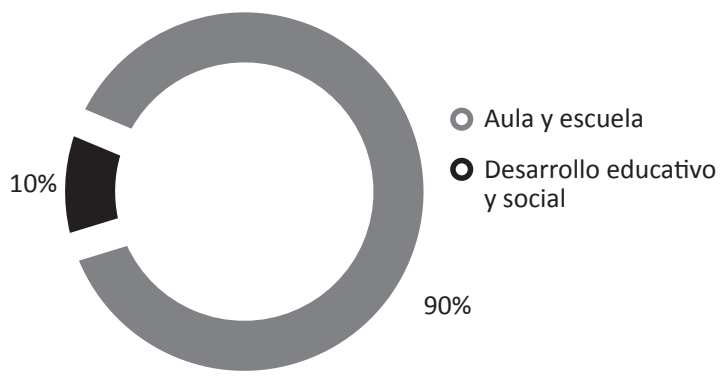

Figura 4. Trabajos de grado del 2011 por líneas de investigación. Elaboración propia. 
Otro hallazgo importante se dio con la aparición de la nueva normatividad - Resolución 36 de mayo de 2010 - hacia el 2010. Gracias a esta, el estudiante podía cursar el primer semestre de estudios de posgrado para suplir la tesis, acorde este procedimiento con los lineamientos en la formación para la educación y en los programas propios ofrecidos por la misma universidad en sus diferentes sedes. Esto permitió encontrar que de los 1316 estudiantes graduados entre 2010 y 2011, 43 estudiantes han tomado la decisión de cursar una especialización o maestría en educación, lo cual representa el $3,2 \%$ de la población.

Sobre las asignaturas y el interés por investigar, en las figuras 5, 6, 7 y 8 se muestran los resultados.

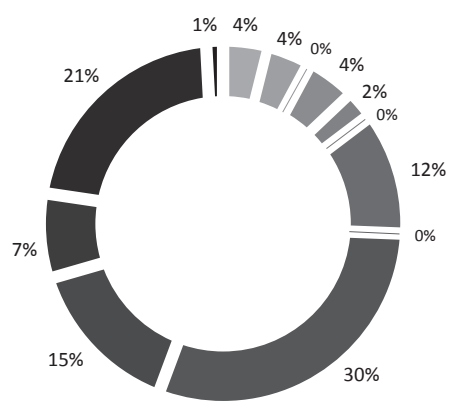

Ciencias naturales
Ciencias sociales
Competencias ciudadanas
Educación artística
Educación física
Educación musical
Ética y valores
Inglés
Lengua castellana
Matemáticas
Multidisciplinar
Pedagogía
Tecnología e informática

Figura 5. Distribución de trabajos de grado por asignaturas en el 2008. Elaboración propia.

El área predominante fue la de Lengua castellana, con un 30\% de los trabajos realizados en este año; le sigue el área de Pedagogía, con un 23\%, y entre las áreas que menos se consulta están Competencias ciudadanas, Inglés, Educación física y Tecnología e informática.

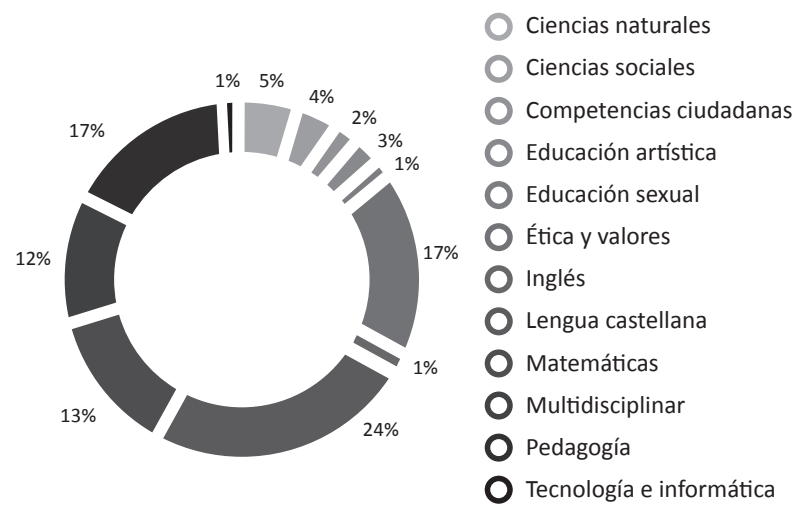

Figura 6. Distribución de trabajos de grado por asignaturas en el 2009. Elaboración propia.
Para el 2009, el mayor porcentaje pertenece a la asignatura de Lengua castellana, que contó con un 24\% de los trabajos realizados; le sigue el área de Pedagogía, con un $17 \%$, y entre las áreas que menos se consultan están Matemáticas, Ciencias naturales, Ciencias sociales, Competencias ciudadanas, Inglés, Educación física y Tecnología e informática con un porcentaje muy bajo respecto a las demás.
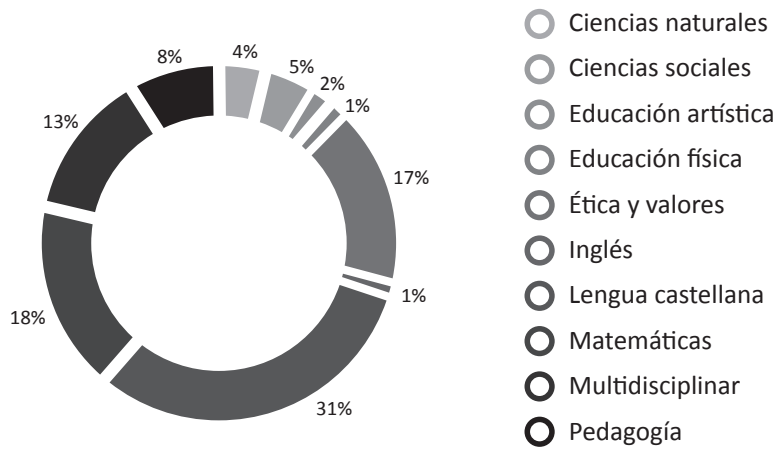

Figura 7. Distribución de trabajos de grado por asignaturas en el 2010. Elaboración propia.

Para el 2010, la asignatura de Lengua castellana obtuvo un 31\% de los trabajos; le sigue el área de Matemáticas, con un $18 \%$, y entre las áreas que menos se consulta están Inglés, Educación física, Educación artística y Ciencias sociales.

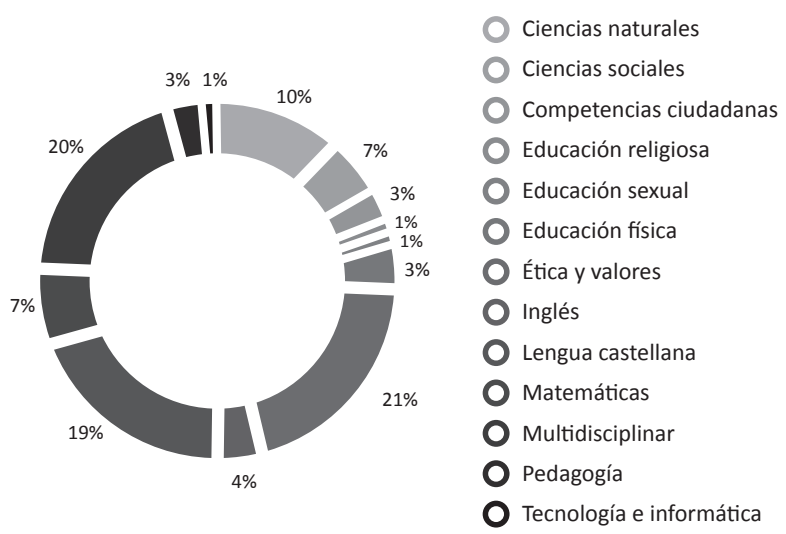

Figura 8. Distribución de trabajos de grado por asignaturas en el 2011. Elaboración propia.

Para el 2011, la asignatura de Ética y valores ocupó el primer lugar, con un $21 \%$ de los trabajos realizados; le sigue el área interdisciplinar, con un 20\%, y el área de Lengua castellana con el 19\%. De igual manera, las áreas en las que menos se investiga están Competencias 
ciudadanas, Inglés, Educación física, Ciencias naturales y Tecnología e informática con un porcentaje muy bajo respecto a los demás.

Se puede decir, de manera general, que los intereses investigativos de los estudiantes de modalidad a distancia, que optaron por escoger los temas relacionados con la asignatura de lengua castellana, fueron los de producción escrita y lectura, semiótica, competencias comunicativas, argumentativas, expositivas, lingüísticas, semántico-comunicativas, procesos de escritura, proceso de abstracción al escribir, gramática, dislexia, disgrafia.

En Matemáticas sobresalieron trabajos de grado cuyos temas se enfocaron en las problemáticas que abarcan las operaciones básicas, el aprendizaje de las matemáticas a través de diferentes actividades como los juegos didácticos y la resolución de problemas, el juego como estrategia para el aprendizaje de las matemáticas, didáctica de las matemáticas, potenciación, radicación, logaritmación, números naturales, competencias matemáticas como productiva, analítica y comunicativa.

En el área de Pedagogía, las tendencias investigativas estuvieron relacionadas con la evaluación educativa y los problemas de deserción escolar. Aquí se encontraron trabajos de grado especiales, como analizar situaciones difíciles de niños con padres en la cárcel.

Por el contrario, se han encontrado muy pocos intereses investigativos en las siguientes asignaturas: en Humanidades (entendida como la asignatura de Inglés), con temas relacionados con la desmotivación hacia el aprendizaje del inglés y la utilización de estrategias didácticas para la enseñanza de una lengua extranjera. En la asignatura Ética, se investigaron temas relacionados con ética, autoridad, respeto, el rescate de los valores, dignidad humana. En Ciencias naturales, los intereses investigativos se centraron en la ecología. En Ciencias sociales, el rescate de la identidad cultural fue el tema de mayor interés, en especial, y tal vez es oportuno decirlo, por el contexto en el que se encuentran las investigaciones realizadas, pues fue importante para reflexionar en la gran riqueza que tiene nuestro país en cuanto a diversidad cultural.

Para la Educación artística, se desarrollaron temáticas relacionadas con la creatividad con el fin de desarrollar habilidades en las diferentes áreas del saber. Se evidenció con esto la interdisciplinariedad como objeto de estudio, pues los estudiantes manifestaron dentro de su trabajo de grado la importancia de innovar y de la creatividad a la hora de desarrollar diversas actividades que se salieran de la monotonía en el aula de clase.
La Educación física se centró en la lúdica como estrategia para promover capacidades y actitudes motivadoras en el aprendizaje de otras áreas. Esta fue utilizada como área interdisciplinar para lograr aprendizajes efectivos en los estudiantes.

Con lo anterior, se puede inferir que los porcentajes de interés de investigación para estas asignaturas fueron bajos con respecto a las asignaturas de Matemáticas, Humanidades y Lengua castellana, tal vez porque no constituyen el área de énfasis del programa, o simplemente porque no componen un objeto de estudio o interés por parte de los estudiantes que se encuentran en un contexto que ofrece problemáticas de aprendizaje en lengua castellana o matemáticas.

\section{Conclusiones}

Finalmente, se puede concluir sobre los resultados presentados en este documento, que los procesos investigativos en la modalidad a distancia dan respuesta positiva a la posibilidad de investigar problemáticas del contexto desde el lugar donde se encuentra el estudiante. Por esta razón, se establece como la opción metodológica que se presenta con mayor frecuencia, es decir, la modalidad de trabajo de grado fue la que presentó mayor porcentaje para desarrollar proyectos de investigación. Esta opción fue preferida por los estudiantes, frente a un mínimo de la escogencia de cursar primer semestre de un programa de posgrado. En su mayoría, los trabajos fueron inscritos en la línea de investigación Aula y Escuela por la pertinencia, coherencia y relevancia frente al tema escogido y porque pueden sentir una mayor inclinación o han preferido por el tipo de comunidad a la cual va dirigida su aplicación, así como de las características propias de la misma investigación que adoptan. Es propio también tener presente los énfasis con los que cuenta el programa, pues de estos surgen muchos problemas por investigar en las diferentes instituciones educativas en los que se desenvuelven los estudiantes y aplican sus trabajos de intervención pedagógica e investigativa.

Se valoró la forma en que se han desarrollado los procesos de investigación en el programa de Licenciatura en Educación Básica con énfasis en Matemáticas, Humanidades y Lengua Castellana. De esta manera, se constituye como un factor predominante a la hora de realizar procesos de autoevaluación y acreditación del mismo, mostrando la calidad investigativa al interior de un programa que es ofrecido en diferentes sedes, 
siendo este un indicador favorable para cualquier institución educativa.

Se evidenció, a través de los cuatro años en que duró la investigación, la relación entre los cambios y la reestructuración de las líneas de investigación del programa, las cuales encaminaron la formación investigativa de los estudiantes de modalidad a distancia. De esta manera, en el momento, se está conformando un interés por continuar con este tipo de investigaciones, para que se dé en forma comparativa en los próximos años y poder así tener otro acercamiento al conocimiento en los diferentes campos investigativos, los cuales repercutirán en mejora de dichos procesos en el resto de las disciplinas encontradas en el programa.

Como consecuencia de este estudio investigativo, se generó una base de datos para que, de manera clara y de rápido acceso, pueda ser estudiada por la comunidad educativa. Además, esta se constituye como una evidencia tangible frente a los procesos de calidad del programa.

Se realizó una aproximación a las experiencias significativas que los estudiantes han realizado con las conclusiones y reflexiones generadas de la aplicación de un trabajo de grado y que han dejando huella en su paso por la universidad. Estas brindaron una mejor comprensión de las problemáticas de la educación, del aprendizaje, de la pedagogía y, en general, del entorno educativo, visto desde diferentes contextos y de diferentes comunidades educativas.

En el mismo sentido, cabe señalar que la investigación realizada por algunos de los integrantes del grupo sIEK permitió reflexionar en torno a que el trabajo desarrollado por los estudiantes de la modalidad a distancia es valioso para los estudiantes, pues deja huella no solo en su vida, sino en la vida de toda una comunidad educativa. Todos estos trabajos se han consolidado, no solo en una estrategia de investigación o de intervención social, sino que, además, construyen conocimiento y comprensión de su entorno educativo. Estos posibilitan que los estudiantes investigadores y sus docentes se adentren en su contexto, conozcan, interpreten y analicen sus prácticas y los fenómenos que ocurren a su alrededor. Por esta razón, merecen un reconocimiento especial de todos aquellos que deseen seguir sus pasos y continuar con el reto que han propuesto.

Con la información analizada, se pretende continuar con otras investigaciones diferentes a las existentes, que se puedan implementar y, por qué no, dar respuesta a los problemas encontrados en las instituciones educativas, de manera que revelen los linea- mientos establecidos en las líneas de investigación del programa de Licenciatura en Educación Básica.

Se sugiere que los programas que se enfocan en la educación, ya sea en la modalidad presencial o a distancia (o mixtas, como se está comenzando a implementar), deben establecer líneas de investigación coherentes, pertinentes y consecuentes con las problemáticas educativas presentadas. De este modo, se propicia el desarrollo investigativo en los estudiantes y que, de una manera reflexiva y crítica, se sitúen en el contexto donde se encuentren, generando a la vez la capacidad de investigar y transformar sus realidades sociales. De esta manera, también se da respuesta a los requerimientos necesarios que demuestren la eficacia ofrecida por las diversas instituciones educativas que están realizando sus procesos de autoevaluación con fines de alcanzar la acreditación de alta calidad.

La formación en investigación para los futuros docentes se debe abordar desde las diversas miradas que abarca la educación. Al mismo tiempo que debe ser analizada a través de una intencionalidad, desde una temática, una población determinada y un contexto que le permita ser estudiada, reflexionada, analizada y dada a conocer a través de las intervenciones de los estudiantes en el aula y que, a su vez, se vea involucrada toda una comunidad educativa.

\section{Referencias}

Arnal, Justo, Delio del Rincón y Antonio Latorre. Investigación educativa: fundamentos y metodologías. Barcelona: Labor, 1994. Impreso.

Consejo Académico Universidad Pedagógica y Tecnológica de Colombia, Tunja, Boyacá. Resolución 36 de 2010. 15 de agosto 2012. Web.

García, Rocío. "Lo que investigan los estudiantes de maestría en educación. Análisis desde un estado del arte”. Boletín virtual REDIPE 820. Enero de 2013.

Gutiérrez, Francisco, y Daniel Prieto. La mediación pedagógica. Buenos Aires: Ediciones Ciccus-La Crujía,1992. Impreso.

Mejía, Marco Raúl, y Amanda Bravo. La sistematización empodera y produce saber y conocimiento. Bogotá: Ediciones desde Abajo, 2008. Impreso.

Messina, Graciela. "Investigación y Experiencia”. Revista Praxis \& Saber 4 (2011): 61-75. Impreso.

Moreno, María Guadalupe. "Potenciar la educación: un currículum transversal de formación para la investigación”. Revista Electrónica Iberoamericana sobre Calidad, Eficacia y Cambio en Educación 3.1 (2005). Impreso. 
Murcia Peña, Napoleón, Napoleón Murcia Gómez y Jesús Ernesto Urbina. "Tendencias investigativas en educación y pedagogía: estudio de las regiones investigativas de la maestría en educación de la uis". Revista Latinoamericana de Estudios Educcativos 7.1 (2011): 81-98. Impreso.

Proyecto Académico Educativo [PAE]. Licenciatura en Educación Básica con énfasis en Matemáticas, Humanidades y Lengua Castellana. Tunja: Escuela de Ciencias Humanísticas y de Educación-FEDAD.UPTC, 2009. Impreso.
Sánchez, Cristina. "Tras las huellas de la investigación educativa”. Nuevos Horizontes pedagógicos 5 (2007). Impreso.

Torres, Alfonso. "La sistematización de experiencias educativas: reflexiones sobre una práctica reciente". Ponencia presentada al Tercer Congreso Iberoamericano y Caribeño de Agentes de Desarrollo Sociocultural y Comunitario, La Habana. 1998. Impreso.

Vélez, Gabriel. "Apuntes iniciales de una búsqueda metodológica para el análisis de procesos de articulación interorganizacionales". Folios 25 (2011): 35. Impreso. 